\section{Introducing genetics: from Mendel to molecule}

\author{
Making sense of the gene \\ Alison Thomas \\ Nelson Thornes Ltd, UK; 2003. 216 pp. \\ $£ 17.00$, paperback. ISBN 0-7487-6440-2 \\ Heredity (2004) 92, 271. doi:10.1038/sj.hdy.6800415 \\ Reviewed by NL Eskeland and NC Bailey
}

Mastering the fundamentals of genetics can be a daunting task for the average reader. In the textbook, 'Introducing Genetics: From Mendel to Molecule,' Dr Alison Thomas describes the field of genetics from its conception to recombinant DNA technology, with a lucid and clear approach.

In Chapter 1, the author introduces the reader to the origins of the study of genetics and the key ideas in the field. In Chapters 2-4, the author succinctly presents the different modes of Mendelian inheritance from monohybrid inheritance to incomplete dominance, $\mathrm{CO}^{-}$ dominance, and dihybrid inheritance, including the test cross and $\chi^{2}$ test statistics. In the section on complementary genes, the author suggests a biochemical explanation for the determination of sweet pea petal color. An example from already-known biochemical reactions would have been more effective in relating how complementary gene products work sequentially.

Chapter 5 explains the chromosome theory of inheritance - that chromosomes are the carriers of Mendel's genes. Nuclear division through mitosis and meiosis are described in depth, followed by revisiting Mendel's laws in explaining Mendel's laws of segregation and independent assortment of alleles. In Chapters 6 and 7, the author describes sex determination and linkage, using crosses and pedigrees; and the usage of test cross in linkage studies and physical mapping to precisely determine the genes' location. In almost every chapter, Dr Thomas meticulously refers back to Mendelian inheritance and its deviations with clarity and simplicity. Chapter 8 addresses chromosome aberrations from two perspectives: how they arise and their genetic and phenotypic consequences. In the first section, the author describes the types of anomalies and their relation to cell division, that is, meiosis 1 and meiosis II. She then discusses a second type of chromosomal aberrations affecting its structure. Thomas uses many cases, both plant and human, to describe the process and results of these abnormalities.

In Chapter 9, Thomas begins with a scenario of a farmer moving from a fertile plateau in the Andes to a new farm on the Patagonian plains to plant his highyielding prize potatoes. Her style is engaging and she, again, cleverly conveys that not all traits obey Mendelian laws in inheritance pattern and expression. Thomas' use of formulas is simple and concise, and her quantitation from a simple mean to the more complex calculation of multifactorial traits is logical and organized. Chapter 10 introduces the reader to principles of genetics and statistics that are applicable to population studies. Topics include definitions used in population genetics, calculating allele frequencies, introducing Hardy-Weinberg law and its derivation from Mendelian principles of inheritance, calculating genotype frequencies, testing equilibrium, disturbances of Hardy-Weinberg equilibrium, which include mutation, migration, genetic drift, and selection. The author goes further to explain in great detail each disturbance using graphs, real photos, and case studies.

Chapters 11 and 12 on heredity at the molecular level is well organized, as Thomas begins describing the genetic code - DNA - and its structure, its replication, transcription, and translation. Each step is clearly developed, concise, and devoid of any elaborative delineation. She ends the section by demonstrating the different types of mutation using two examples - xeroderma pigmentosum and sickle cell anemia. There is no mention, however, of the beneficial effect of mutations such as immunity of sickle cell anemia patients to malaria.

In the final chapter, Thomas introduces the concepts and techniques of recombinant DNA research and its use in both medical and plant biotechnology. Although well written, her descriptions and illustrations are too simplistic and do not convey the complexity involved in genetic engineering. This is shown in her statement 'cattle, sheep and goats are now routinely used as animal biofactories.' Only occasionally such an experiment is successful and without any deleterious result. Furthermore, not all restriction enzymes have sticky ends; hence, more tedious and laborious steps are taken to ensure proper cloning. Nevertheless, her account of the production of transgenic crops is instructive. The table on benefits and risks of GM crops is insightful and a helpful guide in understanding the pros and cons of such technology in plants.

Throughout the book, Thomas' use of real-life examples, charts, and photos clarifies several derivations that would have, otherwise, resulted in ambiguity and confusion. However, for some of the figures, the use of cartoon-like text and drawings should have been avoided for a more professional look. Each chapter ends with a summary, followed by problems to solve (answers at the end of the book), which tests the learner's mastering of the concepts presented. The textbook ends with a glossary of genetics terminology and recommended reading list. Overall, 'Introducing Genetics From Mendel to Molecule,' is a suitable volume for learning the history and fundamentals behind the rapidly evolving field of biotechnology. This is becoming a necessity not only for the biology student, but also for those who are interested in biotechnology.

NL Eskeland, NC Bailey Science2Discover, Inc., PO Box 2435, Del Mar, CA 92014, USA

E-mail: leskeland@science2discover.com 